\title{
PERFORMANCE EVALUATION OF UASBR FOR MILK PROCESSING PLANT OF S.R.THORAT MILK PRODUCTS PVT.LTD AT RAJAPUR
}

\author{
Shashikant.R.Mise ${ }^{1}$, Rajendra Lamkhade ${ }^{2}$ \\ ${ }^{1}$ Professor, Department of Civil Engineering, PDA College of Engineering Gulbarga \\ ${ }^{2}$ AssociateProfessor, Department of Civil Engineering, Pravara Rural College of Engineering Maharashtra \\ srmise45@yahoo.com,rklamkhade@yahoo.com
}

\begin{abstract}
This work was aimed to evaluate the performance of UASBR for treating milk processing wastewater. The study has been conducted for the UASBR installed at S.R.Thorat Milk Products Pvt. Ltd. at Rajapur, Tal. Sangamner, District Ahmednagar. The organic loading rate was $3.69 \mathrm{Kg} \mathrm{COD} / \mathrm{m}^{3} /$ day, hydraulic retention time was 10 hours, and flow rate was $10 \mathrm{~m} 3 / \mathrm{hr}$. And up flow velocity was $0.6 \mathrm{~m} / \mathrm{hr}$. The COD: N: P ratio was followed as 100:5:1 for nutrient feed. The performance of UASBR is reported in terms of \% removal of various wastewater characteristics by UASB process. It is observed that the biogas yield of $0.6 \mathrm{~m}^{3} / \mathrm{m}^{3}$ reactor volume/day is sufficient to carry out natural mixing in the reactor. So Upflow Anaerobic Sludge Blanket (UASB) reactor can be suitable alternatives for treatment of such wastewater to reduce overall operational cost of the treatment. ${ }^{[2]}$
\end{abstract}

Keywords: Wastewater, BOD, COD, Organic Loading Rate (OLR), Milk processing waste.

$* * *$

\section{INTRODUCTION}

The protection and improvement of environment is a major issue, which affects the well being of people and economic development. The rapid urbanization and industrialization is posing additional challenges for environmentalists for proper management of the wastewater, to protect water quality in the receiving water body. In recent years energy consideration and environmental concerns have increased the interest in direct anaerobic treatment of industrial wastes. The anaerobic method of waste treatment offers under the present circumstances a number of significant advantages with little serious or inseparable drawbacks over other treatment methods. The interest in anaerobic liquid wastewater treatment processes in practice is sharply increasing all over the world.

\subsection{Anaerobic Process}

One of the most serious problems in the use of anaerobic treatment for low strength wastes, being the retention of a sufficient quantity of viable sludge under short hydraulic retention time (less than a day) now appears to have been overcome as a result of the development of the high-rate anaerobic processes e.g., anaerobic filters, anaerobic expanded / fluidized bed reactor, and Upflow Anaerobic Sludge Blanket (UASB) reactor. ${ }^{[4]}$ One common feature offered by all the high-rate processes is the ability to achieve high solids retention time, even at short hydraulic retention time. In anaerobic filters and expanded / fluidized beds, this is accomplished by the development of biofilm on support surfaces. ${ }^{[5]}$

\subsection{Sources of Wastewater}

- Process waste streams: Milk processing wastewater

- Non-process waste streams: Cooling water, Waste wash water, Water treatment plant wastewater, Boiler blow down, Bottling plant wash wastewater, other wastes.

\subsection{Objectives of the Study}

The study was conducted at S.R.Thorat Milk Products Pvt. Ltd., Rajapur, Sangamner, Ahmednagar, and Maharashtra with the following objectives.

1) To determine characteristics of Milk processing industry wastewater.

2) To study the efficiency of the UASBR.

3) To study the anaerobic digestion under optimum conditions.

4) To find the amount of biogas produced.

\section{MATERIALS AND METHODOLOGY}

\subsection{Analysis of General Characteristics}

$2000 \mathrm{ml}$ of effluent sample was collected for analysis of various physico-chemical parameters such as $\mathrm{pH}$, BOD, COD, alkalinity, total solids, etc. and the analysis were carried out as per the Standard Methods, $20^{\text {th }}$ edition. ${ }^{[1]}$

\section{Process Design Parameters:-}

$>$ Hydraulic Retention Time (HRT): 10 hours. 
$>$ Solids Retention Time (SRT): 30 days.

$>$ Organic Loading on sludge blanket: $3.69 \mathrm{~kg}$ COD $/ \mathrm{m}^{3} /$ day.

$>$ Flow Regime : Constant rate for pumped inflows

$>$ Upflow velocity: $0.6 \mathrm{~m} /$ hour.

$>$ Flow rate: $10 \mathrm{~m}^{3} /$ hour.

$>$ COD:N:P ratio : 100:5:1

$>$ Gas production : $60 \mathrm{~m}^{3} /$ day

$>$ Inlet feed points: 2 Nos.

\section{RESULTS AND DISCUSSION}

\subsection{Characteristics of Wastewater:}

The fresh wastewater samples were brought to laboratory to analysis the typical characteristics. The key pollutants in the wastewater from the milk processing industry are organic compounds and solids. Biodegradability may be estimated on the basis of BOD / COD ratio. This ratio ranges between 0.210.23 , which indicates that the part of the organic compounds in the wastewater is not easily biodegradable. The $\mathrm{BOD}_{5}$ is $534 \mathrm{mg} / \mathrm{L}$, COD is $2,461 \mathrm{mg} / \mathrm{L}$, Total Dissolved Solids in wastewater was found to be $2382 \mathrm{mg} / \mathrm{L}$ and Total Suspended Solids was $488 \mathrm{mg} / \mathrm{L}$. The $\mathrm{pH}$ value in this case varies from 7.4 - 7.6. When wastewater contains high concentration of organic matter, dissolved oxygen depletes because of the breakdown of organic matter, in absence of oxygen, sulphate acts as an electron acceptor to produce $\mathrm{H}_{2} \mathrm{~S}$ and odour. The results of $\mathrm{pH}$, alkalinity, percentage COD removal, Gas production are shown in Table no.1, Table no. $2 \&$ Table no.3.

Table1. Influent Waste Water Characteristics

\begin{tabular}{|c|c|c|c|c|c|c|c|c|c|c|c|c|}
\hline $\begin{array}{c}\text { Sample } \\
\text { No. }\end{array}$ & $\begin{array}{c}\text { Time } \\
\text { (Days) }\end{array}$ & $\begin{array}{c}\text { Temp. } \\
{ }^{\circ} \mathrm{C}\end{array}$ & pH & $\begin{array}{l}\text { TDS } \\
\mathrm{mg} / \mathrm{L}\end{array}$ & $\begin{array}{c}\text { TSS } \\
\mathrm{mg} / \mathrm{L}\end{array}$ & $\begin{array}{l}\text { BOD } \\
\mathrm{mg} / \mathrm{L}\end{array}$ & $\begin{array}{l}\text { COD } \\
\mathrm{mg} / \mathrm{L}\end{array}$ & $\begin{array}{c}\mathrm{Cl} \\
\mathrm{mg} / \mathrm{L}\end{array}$ & $\begin{array}{c}\mathrm{SO}_{4} \\
\mathrm{mg} / \mathrm{L}\end{array}$ & $\begin{array}{l}\text { TKN } \\
\mathrm{mg} / \mathrm{L}\end{array}$ & $\begin{array}{l}\text { O\&G } \\
\mathrm{mg} / \mathrm{L}\end{array}$ & $\begin{array}{l}\text { VFA } \\
\mathrm{mg} / \mathrm{L}\end{array}$ \\
\hline 1 & 1 & 26 & 7.5 & 2400 & 486 & 510 & 2448 & 190 & 450 & 32.06 & 9 & 94 \\
\hline 2 & 4 & 26 & 7.5 & 2360 & 500 & 580 & 2544 & 190 & 445 & 31.78 & 8 & 81 \\
\hline 3 & 8 & 26 & 7.4 & 2390 & 476 & 590 & 2368 & 190 & 451 & 31.92 & 8 & 81 \\
\hline 4 & 11 & 25 & 7.5 & 2340 & 468 & 470 & 2272 & 190 & 448 & 31.64 & 7 & 77 \\
\hline 5 & 15 & 24 & 7.6 & 2410 & 512 & 600 & 2576 & 192 & 440 & 31.64 & 8 & 81 \\
\hline 6 & 18 & 25 & 7.4 & 2420 & 490 & 540 & 2496 & 194 & 441 & 31.78 & 9 & 94 \\
\hline 7 & 22 & 26 & 7.5 & 2390 & 470 & 470 & 2336 & 194 & 430 & 32.06 & 8 & 94 \\
\hline 8 & 25 & 25 & 7.5 & 2340 & 488 & 520 & 2464 & 194 & 435 & 31.92 & 8 & 90 \\
\hline 9 & 29 & 27 & 7.5 & 2350 & 508 & 590 & 2560 & 192 & 425 & 32.20 & 8 & 94 \\
\hline 10 & 32 & 25 & 7.6 & 2330 & 498 & 550 & 2528 & 196 & 420 & 32.06 & 8 & 81 \\
\hline 11 & 36 & 27 & 7.5 & 2360 & 478 & 490 & 2384 & 194 & 434 & 32.06 & 9 & 86 \\
\hline 12 & 39 & 27 & 7.5 & 2380 & 480 & 490 & 2416 & 190 & 443 & 32.20 & 8 & 86 \\
\hline 13 & 43 & 28 & 7.6 & 2390 & 492 & 570 & 2528 & 190 & 449 & 31.92 & 8 & 86 \\
\hline 14 & 46 & 28 & 7.6 & 8370 & 472 & 470 & 2320 & 190 & 439 & 32.06 & 9 & 90 \\
\hline 15 & 50 & 27 & 7.6 & 2420 & 474 & 480 & 2336 & 194 & 446 & 32.06 & 9 & 94 \\
\hline 16 & 53 & 29 & 7.5 & 2410 & 482 & 500 & 2432 & 194 & 434 & 31.78 & 8 & 90 \\
\hline 17 & 57 & 29 & 7.5 & 2400 & 486 & 510 & 2448 & 192 & 432 & 31.78 & 7 & 90 \\
\hline 18 & 60 & 27 & 7.5 & 2400 & 494 & 570 & 2528 & 192 & 445 & 31.78 & 8 & 81 \\
\hline 19 & 64 & 32 & 7.6 & 2410 & 490 & 530 & 2480 & 190 & 449 & 32.06 & 8 & 81 \\
\hline 20 & 67 & 29 & 7.4 & 2430 & 504 & 590 & 2560 & 194 & 420 & 32.20 & 8 & 81 \\
\hline 21 & 71 & 29 & 7.5 & 2390 & 504 & 590 & 2560 & 194 & 427 & 32.06 & 7 & 81 \\
\hline 22 & 74 & 29 & 7.5 & 2370 & 496 & 560 & 2528 & 194 & 430 & 32.06 & 8 & 90 \\
\hline 23 & 78 & 30 & 7.6 & 2370 & 492 & 550 & 2512 & 192 & 430 & 31.92 & 8 & 90 \\
\hline 24 & 81 & 32 & 7.5 & 2360 & 488 & 510 & 2448 & 192 & 439 & 32.06 & 9 & 77 \\
\hline
\end{tabular}




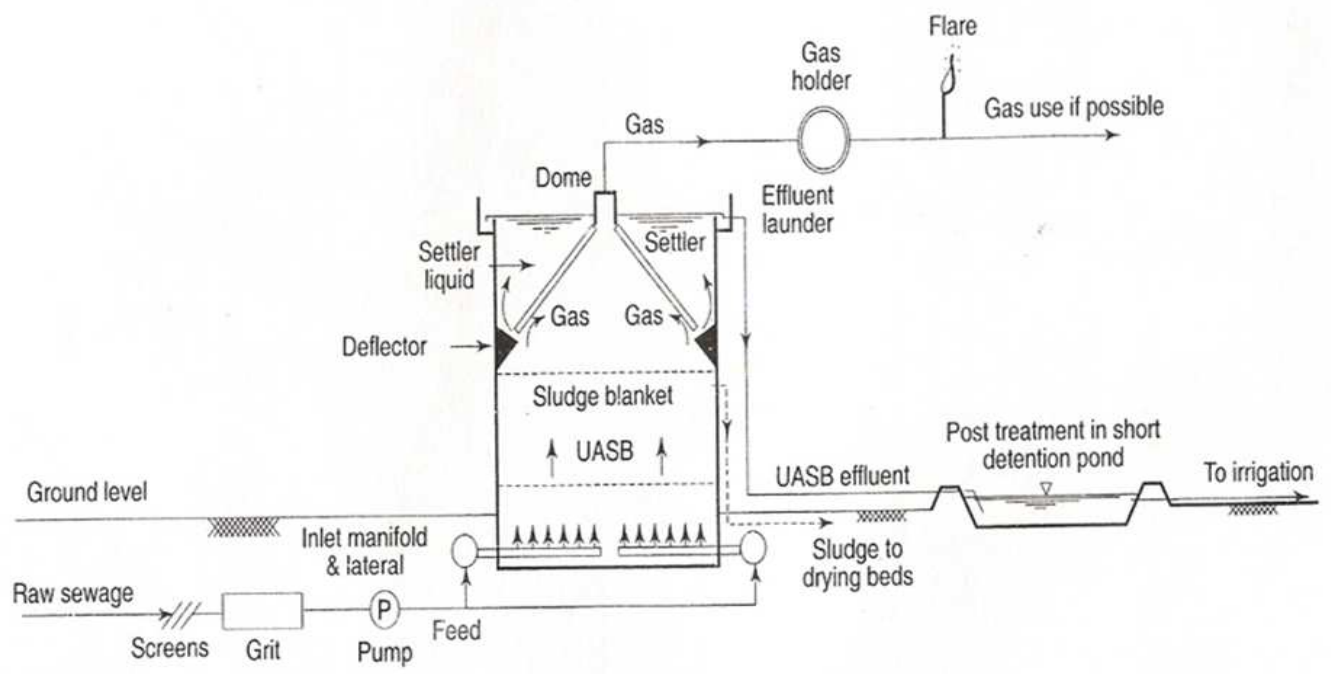

Fig1. A UASBR installation for Milk Processing Wastewater

Table2. Effluent Waste Water Characteristics

\begin{tabular}{|c|c|c|c|c|c|c|c|c|c|c|c|c|}
\hline $\begin{array}{c}\text { Sample } \\
\text { No. }\end{array}$ & $\begin{array}{c}\text { Time } \\
\text { (Days) }\end{array}$ & $\begin{array}{c}\text { Temp. } \\
{ }^{\circ} \mathrm{C}\end{array}$ & pH & $\begin{array}{c}\text { TDS } \\
\mathrm{mg} / \mathrm{L}\end{array}$ & $\begin{array}{c}\text { TSS } \\
\mathrm{mg} / \mathrm{L}\end{array}$ & $\begin{array}{l}\text { BOD } \\
\mathrm{mg} / \mathrm{L}\end{array}$ & $\begin{array}{l}\text { COD } \\
\mathrm{mg} / \mathrm{L}\end{array}$ & $\begin{array}{c}\mathrm{Cl} \\
\mathrm{mg} / \mathrm{L}\end{array}$ & $\begin{array}{c}\mathrm{SO}_{4} \\
\mathrm{mg} / \mathrm{L}\end{array}$ & $\begin{array}{l}\text { TKN } \\
\mathrm{mg} / \mathrm{L}\end{array}$ & $\begin{array}{l}\text { O\&G } \\
\mathrm{mg} / \mathrm{L}\end{array}$ & $\begin{array}{l}\text { VFA } \\
\mathrm{mg} / \mathrm{L}\end{array}$ \\
\hline 1 & 1 & 28 & 7.3 & 1350 & 80 & 70 & 240 & 190 & 148 & 61.74 & 0 & 17.1 \\
\hline 2 & 4 & 28 & 7.3 & 1320 & 76 & 74 & 256 & 190 & 144 & 61.74 & 0 & 8.5 \\
\hline 3 & 8 & 28 & 7.3 & 1340 & 78 & 68 & 240 & 190 & 152 & 62.86 & 0 & 8.5 \\
\hline 4 & 11 & 27 & 7.3 & 1310 & 80 & 66 & 224 & 190 & 144 & 62.86 & 0 & 8.5 \\
\hline 5 & 15 & 27 & 7.4 & 1360 & 72 & 74 & 256 & 192 & 140 & 62.58 & 0 & 12.8 \\
\hline 6 & 18 & 28 & 7.3 & 1370 & 76 & 70 & 240 & 194 & 140 & 62.02 & 0 & 12.8 \\
\hline 7 & 22 & 28 & 7.2 & 1390 & 70 & 68 & 224 & 194 & 132 & 61.88 & 0 & 8.5 \\
\hline 8 & 25 & 28 & 7.3 & 1290 & 78 & 70 & 240 & 194 & 132 & 62.02 & 0 & 17.1 \\
\hline 9 & 29 & 29 & 7.3 & 1280 & 72 & 74 & 256 & 192 & 128 & 62.16 & 0 & 12.8 \\
\hline 10 & 32 & 28 & 7.3 & 1270 & 76 & 72 & 256 & 196 & 128 & 62.30 & 0 & 12.8 \\
\hline 11 & 36 & 30 & 7.3 & 1280 & 78 & 68 & 240 & 194 & 136 & 62.16 & 0 & 12.8 \\
\hline 12 & 39 & 29 & 7.3 & 1290 & 68 & 68 & 240 & 190 & 144 & 62.02 & 0 & 8.5 \\
\hline 13 & 43 & 30 & 7.4 & 1300 & 70 & 72 & 256 & 190 & 148 & 62.16 & 0 & 8.5 \\
\hline 14 & 46 & 30 & 7.4 & 1320 & 72 & 68 & 224 & 190 & 140 & 61.74 & 0 & 17.1 \\
\hline 15 & 50 & 29 & 7.4 & 1310 & 72 & 68 & 224 & 194 & 144 & 61.74 & 0 & 17.1 \\
\hline 16 & 53 & 31 & 7.3 & 1400 & 78 & 70 & 240 & 194 & 132 & 62.72 & 0 & 12.8 \\
\hline 17 & 57 & 31 & 7.3 & 1400 & 76 & 70 & 240 & 192 & 132 & 62.58 & 0 & 12.8 \\
\hline 18 & 60 & 31 & 7.3 & 1390 & 78 & 72 & 256 & 192 & 144 & 62.22 & 0 & 8.5 \\
\hline 19 & 64 & 32 & 7.35 & 1400 & 74 & 70 & 240 & 190 & 148 & 62.44 & 0 & 12.8 \\
\hline 20 & 67 & 32 & 7.2 & 1410 & 78 & 74 & 256 & 194 & 128 & 62.72 & 0 & 12.8 \\
\hline 21 & 71 & 31 & 7.3 & 1440 & 72 & 74 & 256 & 194 & 128 & 62.72 & 0 & 8.5 \\
\hline 22 & 74 & 32 & 7.25 & 1410 & 72 & 72 & 256 & 194 & 132 & 63.00 & 0 & 8.5 \\
\hline 23 & 78 & 32 & 7.45 & 1390 & 76 & 72 & 256 & 192 & 132 & 63.00 & 0 & 12.8 \\
\hline 24 & 81 & 33 & 7.3 & 1390 & 80 & 70 & 240 & 192 & 140 & 62.44 & 0 & 12.8 \\
\hline
\end{tabular}


Table3 Performance of UASB Reactor

\begin{tabular}{|c|c|c|c|c|c|c|c|c|}
\hline \multirow{2}{*}{$\begin{array}{c}\text { Sample } \\
\text { No. }\end{array}$} & \multirow{2}{*}{$\begin{array}{c}\text { Time } \\
\text { (Days) }\end{array}$} & \multicolumn{7}{|c|}{ Percentage Removal } \\
\hline & & TDS & TSS & BOD & COD & $\mathrm{SO}_{4}$ & O\&G & VFA \\
\hline 1 & 1 & 43.75 & 83.54 & 86.27 & 90.19 & 67.11 & 100 & 81.81 \\
\hline 2 & 4 & 44.40 & 84.40 & 87.24 & 89.94 & 66.15 & 100 & 89.51 \\
\hline 3 & 8 & 43.93 & 85.71 & 88.47 & 89.89 & 66.30 & 100 & 89.51 \\
\hline 4 & 11 & 44.01 & 85.89 & 85.96 & 90.14 & 67.85 & 100 & 88.96 \\
\hline 5 & 15 & 43.56 & 85.54 & 87.67 & 90.06 & 68.18 & 100 & 84.19 \\
\hline 6 & 18 & 43.39 & 84.49 & 87.04 & 90.38 & 68.25 & 100 & 86.38 \\
\hline 7 & 22 & 41.84 & 85.11 & 85.53 & 90.41 & 69.30 & 100 & 90.95 \\
\hline 8 & 25 & 44.87 & 84.01 & 86.45 & 90.25 & 69.65 & 100 & 80.22 \\
\hline 9 & 29 & 45.53 & 85.83 & 87.45 & 90.00 & 69.88 & 100 & 86.38 \\
\hline 10 & 32 & 45.49 & 84.34 & 86.91 & 89.87 & 69.52 & 100 & 84.19 \\
\hline 11 & 36 & 45.76 & 83.68 & 86.12 & 89.93 & 68.66 & 100 & 85.12 \\
\hline 12 & 39 & 45.80 & 85.83 & 86.12 & 90.06 & 67.49 & 100 & 90.12 \\
\hline 13 & 43 & 45.61 & 85.77 & 87.37 & 89.87 & 67.04 & 100 & 90.12 \\
\hline 14 & 46 & 44.30 & 84.75 & 85.53 & 90.34 & 68.11 & 100 & 81.00 \\
\hline 15 & 50 & 45.86 & 84.81 & 85.53 & 90.41 & 67.71 & 100 & 81.81 \\
\hline 16 & 53 & 41.91 & 83.82 & 86.00 & 90.13 & 66.82 & 100 & 85.77 \\
\hline 17 & 57 & 41.67 & 84.36 & 86.27 & 90.19 & 69.44 & 100 & 85.77 \\
\hline 18 & 60 & 42.08 & 84.21 & 87.72 & 89.87 & 67.64 & 100 & 89.51 \\
\hline 19 & 64 & 41.91 & 84.90 & 86.42 & 90.32 & 67.04 & 100 & 84.20 \\
\hline 20 & 67 & 41.97 & 84.52 & 87.46 & 90.00 & 69.52 & 100 & 84.20 \\
\hline 21 & 71 & 39.75 & 84.71 & 87.46 & 90.00 & 72.02 & 100 & 89.50 \\
\hline 22 & 74 & 40.51 & 85.48 & 87.14 & 89.87 & 69.30 & 100 & 90.55 \\
\hline 23 & 78 & 41.35 & 84.46 & 86.91 & 89.81 & 69.30 & 100 & 85.77 \\
\hline 24 & 81 & 41.10 & 83.61 & 86.27 & 90.19 & 69.12 & 100 & 83.37 \\
\hline
\end{tabular}

\subsection{Discussion}

TSS removal was in the range of 82 to $86 \%$, the BOD reduction was achieved in the range of 84 to $89 \%$, the COD reduction observed was 88 to $91 \%$, the VFA got reduced by 80 to $90 \%$, the sulphates removal was 66 to $72 \%$ and $100 \%$ removal of oil and grease was observed. These values of the percentage removal of various parameters are in itself an indicator of the efficient and good performance of the UASB reactor at S.R.Thorat Milk Products Pvt. Ltd., These values are also indicative of the stability of the UASB reactor.

\section{CONCLUSIONS}

1. At the milk processing plant it has been observed that the production of methane gas on an average is about $60 \mathrm{~m}^{3} /$ day with a methane content of $75 \%$. This amounts to a comparative revenue generation of Rs. $1,37,970=00$ per annum.

2. The fact that the UASB process generates energy rather than consuming it, amounts to positive environmental impact.

3. Wherever the UASB process is feasible to provide, it is the least demanding process on resources in terms of land, energy, operating \& maintenance costs. The land requirement is just above $5 \%$ of that of most of the conventional treatment processes.

4. The installation \& operational cost for the $100 \mathrm{~m}^{3}$ UASB reactor at S.R. Thorat Milk Products Pvt. Ltd. has been realized as Rupees One Crore (Rupees Ten Million).

5. The maximum COD efficiency observed is $91 \%$. 


\section{REFERENCES}

[1] Lettinga B. Hulshoff Pol. W. 1991 : UASB process design for various types of wastewaters (J), Wat. Sci.Technology, - 24(8); 87.

[2] Handa, B.K. and Rajesh Seth (1990) Waste Management in distillery industry. Journal IAEM, 17, 44-54.

[3] America Public Health Association (APHA) Standard Methods for the Examination of Water and Wastewater, Washington, D.C. $\left(20^{\text {th }}\right.$ edition $)$

[4] Ghangrekar .M.M. Kahalekar JU., and Sakle J.J. (2002) Applicable loading rates for design of Upflow Anaerobic Sludge Blanket (UASB) reactor, J. of Institution of Enginers (India), 82, 48-53.

[5] Bal A.S. and Dhagat N.N. (201) Upflow anaerobic sludge blankets a review. Indian J. of Environmental Health, 43(2), 1-82. 